\title{
On The Pragmatic Content of Science and Common Sense
}

Roberto Gronda and Giacomo Turbanti

\section{CpenEdition}

1 Journals

Electronic version

URL: http://journals.openedition.org/ejpap/1068

DOI: 10.4000/ejpap.1068

ISSN: 2036-4091

\section{Publisher}

Associazione Pragma

\section{Electronic reference}

Roberto Gronda and Giacomo Turbanti, « On The Pragmatic Content of Science and Common Sense », European Journal of Pragmatism and American Philosophy [Online], IX-2 | 2017, Online since 22 January 2018, connection on 19 April 2019. URL : http://journals.openedition.org/ejpap/1068 ; DOI : 10.4000/ ejpap.1068

This text was automatically generated on 19 April 2019

\section{(i) $(9$}

Author retains copyright and grants the European Journal of Pragmatism and American Philosophy right of first publication with the work simultaneously licensed under a Creative Commons AttributionNonCommercial-NoDerivatives 4.0 International License. 


\title{
On The Pragmatic Content of Science and Common Sense
}

\author{
Roberto Gronda and Giacomo Turbanti
}

\section{Introduction}

1 The relationship between science and common sense has traditionally been a major concern in the history of pragmatist philosophy. Starting from Dewey, pragmatists have devoted great deal of attention to the ways in which science and common sense can interact, their proposal being that of defusing the possible elements of conflict between what they conceived as two attitudes towards the world. In the fourth chapter of his Logic: Theory of Inquiry, Dewey identified common sense with those situations in which "human beings are directly involved" (common sense world), as well as with the "inquiries that take place in making the required adjustments in behavior" (common sense inquiries) (Dewey 1938: 66). Common sense inquiries, he argued, differ from scientific ones in that the former are "concerned with qualitative matter and operations," while the meanings and significances that are used in the latter are determined "on the ground of their systematic relations of coherence and consistency with one another," that is, they are intra-theoretically defined (Dewey 1938: 71). In doing so, Dewey did not conceive of science and common sense as opposites, but rather as two different and equally legitimate ways of framing and dealing with ultimately practical problems. Consequently, he completely eschewed the widespread idea of the intrinsic conflict between science and common sense, thus making room for a plurality of approaches to the world.

2 The goal of our paper is to revisit and refresh such a pragmatist, pluralist insight. Our analysis will follow two distinct, yet strictly interrelated paths. On the one hand, it will take into account the structure of two normative spaces - one in the framework of common sense, the other in the framework of science - in order to highlight their different principles of constitution of objectivity. By adapting Wilfrid Sellars' terms, we will call these two normative spaces, respectively, the manifest image and the scientific image, and we will hold that the difference in their structures can be expressed in terms 
of the different kinds of inferences that the two normative spaces allow us to make. ${ }^{1}$ Such an approach, which may be called structural, emphasizes the differences between science and common sense rather than their continuity. It provides us with a picture that somehow crystallizes into a static 'essence' (the structure) the outcome of a long and complex process of conceptual refinement. Since we believe inferences can be analyzed as sets of sentences - that is, inferences can be treated as relations between linguistic sentences without loss of explanatory import - the investigation into the normative structures of common sense and science can be profitably conceived of as an attempt to reconstruct and clarify the different languages - or vocabularies, in Rortyian terms - of the manifest and the scientific image.

3 On the other hand, our analysis will also focus on the concrete practices that underlie the languages of science and common sense, and make it possible for us to use them properly. We take concepts to be determined by the ways in which they are applied in certain normative practices. Of course the application of concepts in judgments is part of these practices. In this sense, we say that linguistic expressions have a pragmatic content that is grounded on certain normative practices. Contrary to the linguistic, structural account of the manifest and the scientific image, the analysis of manifest and scientific practices results in a more continuous picture of the relationship between the two normative spaces. Such a picture preserves the platitudinous intuition that science did not come out of nowhere. Historically speaking, indeed, there is a strong continuity between mechanical arts and the methods of scientific research: scientific practice is commonly seen as a refinement and amelioration of the tools and crafts of artisans. It is worth noting, however, that that process of refinement does not simply come down to a technical improvement of the instruments that come to be adopted in the scientific practice. The relevant point is rather that, by being inserted into a new context, i.e., the laboratory, the tools and crafts of artisans undergo a process of recharacterization that goes hand in hand with the elaboration of new standards and new ends that rule scientific practice. Dewey depicted the relation between the two kinds of practices as a shift from empirical experience to experimental experience. ${ }^{2}$ Consequently, our insistence on the continuity between manifest and scientific practices does not flatten the differences that exist between the two; rather, it places them in a historical and material continuum that makes it possible to tell a story about the genesis of the scientific image from the manifest image. This is similar to what Dewey had in mind when sketching a natural history of logical thinking. We believe that the adoption of a perspective of this sort enhances the explanatory power of our account.

As is well known, in recent years much has been written on the "language versus experience" debate. ${ }^{3}$ Scholars more inclined towards classical pragmatism have privileged the latter over the former, while contemporary pragmatists have stuck to the linguistic turn, and have suggested jettisoning the concept of experience because it lacks clarity. However, as Mark Johnson has correctly remarked, it is obvious that "any strong contrast between experience and language is just one more big dichotomy" (Johnson 2014: 14); consequently, this way of framing the issue is unwarranted from a pragmatist perspective. Our account is in accord with Johnson's line of reasoning, and in doing so it aims to preserve the best of both approaches: if asked whether we privilege language over practice (we take "practice" as synonymous with "experience"), or viceversa, our answer would be "Both!" We take language and practice to be two aspects of a broader and encompassing whole, namely a normative space like the scientific and the manifest 
image. Mimicking Kant, it may therefore be said that, within the scientific or the manifest image, language without practice is empty, whereas practice without language is blind. ${ }^{4}$

The three main sections that make up the present article will be devoted to investigating the different relationships that hold between science and common sense. In the next section we will introduce the topic by presenting Sellars' well-known distinction between the manifest and the scientific image. Sellars' analysis is by far the most influential account of the relationship between science and common sense. We rely on his distinction to develop our own analytical tools, which we will use to clarify the kind of relation between science and common sense that interest us. First, we take this relation into account at the level of language. Then in section 3, that same relation is investigated at the level of practices. Our goal is to articulate the pragmatist insight that the ability to apply conceptual contents is grounded on normative practices. Finally in section 4 , we utilize Brandom's meaning-use analysis to systematize the complex net of relations between science and common sense highlighted in the two previous sections.

\section{Ways of Representing the World}

6 An iconic picture of the complex interrelation between science and common sense was drawn by Wilfrid Sellars in his now classic Philosophy and the Scientific Image of Man (1962), where he introduced the distinction between the scientific image and the manifest image. Both images are ideal types in which complete representations of the world are provided. In the manifest image the world is commonsensically represented "as we encounter it in perception and self-awareness" (Sellars 1962: 14); objects have intrinsic properties that determine their behavior. The way in which Sellars characterizes the ontology of the manifest image is quite interesting: he argues that its primary objects can be thought of in terms of the category of person. He suggests that the manifest image can be described as the categorial refinement of an original, mythical image in which all objects are in fact treated as real persons and all events are explained as intentional actions. So, for instance, in order to explain why the wind took off the hut one could answer "because it is angry at us," or "because it intended to push clouds away and it didn't notice us." The categories of the manifest image would then derive from such original image through a process "of a gradual pruning of the implications of saying with respect to what we would call an inanimate object, that it did something" (Sellars 1962: 12): in the manifest image, objects do not have intentions and do not act, but their behavior is still explained in terms of their character or nature. In other words, in the manifest image the correlations between properties and events that explain the behavior of things in the world are thought of in terms of the powers and dispositions of person-like objects. In order to exemplify what he has in mind, Sellars often refers to Aristotle's theory of being and substance (Sellars 1975: I.29): the nature of a substance can be thought of as its form or essence. In a sense, the manifest image is clearly characteristic of common sense. ${ }^{5}$ Nonetheless, the manifest image is quite "scientifically sophisticated" as far as its empirical and conceptual resources are concerned. So, for instance, Sellars includes in the manifest image complex inductive methods such as statistical inference, which can be used to investigate correlations between properties of things in the world. What definitionally distinguishes the manifest from the scientific image, for Sellars, is the fact that only in the latter we are allowed to use postulational methods: while explanations in the manifest image purely correlate manifest properties of manifest objects, explanations 
in the scientific image involve "the postulation of imperceptible entities" (Sellars 1962: 7). In order to highlight this distinction, Sellars suggests thinking about the difference between two kinds of explanation for the fact that a balloon has expanded, the one in terms of the Boyle-Charles law for an ideal gas, the other in terms of the kinetic theory of gases (Sellars 1956: 150). The first one establishes a correlation between manifest properties of gases: if the temperature remains constant, the pressure exerted by the mass of the gas is inversely proportional to its volume. The second one postulates imperceptible particles, atoms and molecules, which the gas consists of, so that the properties of the latter can be accounted for in terms of the behavior of these particles as defined by the kinetic laws that govern their motion: pressure consists of the particle hitting the surface of the container of the gas, so - as long as the kinetic energy of the particle is held constant - if the volume of the container is smaller, then the frequency with which the particles hit its surface is higher. Notice that the kind of explanation provided in the scientific image strips away the ontology of the manifest image: as Sellars points out, "it is because a gas is - in some sense of 'is' - a cloud of molecules which are behaving in certain theoretically defined ways, that it obeys the empirical Boyle-Charles law" (Sellars 1961: 121).

7 A few remarks are necessary at this point, in order to explain how we intend to build on these Sellarsian materials. There are two aspects of Sellars' account that, at least for our purposes, need further elaboration. First, we accept the distinction between the manifest and the scientific image in terms of the notion of postulation, but we believe that it requires some qualification because Sellars never really gives an explicit analysis of it. Second, the distinction between the two images was originally introduced by Sellars in relation to the problem of discussing the clash between the manifest concept of man as a rational subject of intentional states and the (at the time mainly non-cognitivist) scientific concept of man as a complex system of physical, physiological and neurophysiological states. In this sense both the manifest and the scientific image are thought by Sellars as images of man-in-the-world. The fortune of the Sellarsian distinction, however, corresponds to a somewhat more liberal use of it: the two images have been taken as images of the world. Following such a use, we will exploit the distinction between the two ideal types of the manifest and the scientific image as a technical tool for the investigation of the more general frameworks of science and common sense. In order to do so, we introduce the labels MI and SI to designate these two technical notions - the normative spaces of the manifest and the scientific image respectively.

8 Since our use of the notions of MI and SI does not coincide with Sellars' original one we should say a few more words about it. In the first place, it is worth emphasizing that by $\mathrm{MI}$ and SI we refer to normative spaces. A normative space determines the conditions for concept application, in the sense that the way in which conceptual contents can be articulated and ultimately determined depends on the structure of a normative space. A collection of concepts that can be coherently applied together forms a conceptual repertoire. Therefore, a conceptual repertoire is not a normative space; rather, a conceptual repertoire is possible only within a normative space, and the same normative space can accommodate different conceptual repertoires. Similarly, a normative space must be carefully distinguished from the representation of things in the world that can be provided in terms of a conceptual repertoire.

9 In the second place, it is equally important to remark that, even if we utilize the notions of MI and SI to discuss the relations between common sense and science, MI does not 
entirely coincide with common sense, nor does SI entirely coincide with science. Both MI and SI stem from a theoretical refinement of some elements of the two frameworks. Science, for instance, is much more complicated than SI. Actual, concrete science is not merely postulational: part of the scientific activity in effect consists of searching for empirical correlations, and thus it belongs to MI. Moreover, the sociology of science has shown how many external factors take part in the process of constitution of scientific objectivity (Latour \& Woolgar 1979). As a consequence of the practical turn in philosophy of science, we can no longer conceive of all those factors as something merely external to scientific activity, a kind of scaffolding that supports scientific research without interfering with it. The same holds true for the distinction between MI and common sense. To give only an example, the latter has a moral and aesthetic dimension which is completely lacking in MI.

10 At this point, however, a possible objection should be addressed. One might wonder why we choose to focus on these normative spaces if our purpose is to discuss the relation between science and common sense. The answer is methodological and theoretical at the same time. It is sensible for anyone preparing for an investigation in this area to acknowledge that science and common sense are both heavily overloaded categories: they are deployed in so many diverse fields and characterized in so many different ways that it is almost impossible to simply call them up without engendering anything but confusion. Thus, our dealing with MI and SI is expedient to mark off more precisely the topic of our analysis: such topic is the comparison between the pragmatic content of science and common sense. We employ the notion of 'pragmatic content' to refer to the fact that linguistic contents are grounded on normative practices. ${ }^{6} \mathrm{In}$ fact, we believe that our characterization of MI and SI highlights just a few of the distinctive features that are essential to the more general frameworks of common sense and science respectively.

11 All this being said, we must now finally clarify the postulational nature of SI. Following Sellars, we define the distinction between MI and SI in terms of the postulational explanatory methods that are allowed in the latter but not in the former. The activity that is distinctive of MI is the search for empirical correlations. On the contrary, SI revolves around the act of postulating entities to explain why MI objects are subjected to those MI correlations. According to this characterization, any explanation that is based on a framework of postulated entities presents itself as a candidate for SI. ${ }^{7}$ It is worth noting, however, that the relevant qualification is not much sheer postulation, but rather the construction of an explanatory framework in which the postulated entities account for the behavior of MI objects without recourse to their powers and dispositions.

12 It may be useful here to distinguish between two different forms of postulation: 'spurious' and 'genuine' postulation. So, for instance, one could try to explain why opium makes one sleep by postulating a virtus dormitiva. This is clearly an instance of spurious postulation. Indeed, the postulation of the opium's sleeping power does not qualify as a scientific explanation in SI. Notoriously, the problem with the virtus dormitiva is that it has no real explanatory power. Notice however that there are examples of spurious postulation that in effect provide satisfactory explanations, even if they do not qualify for SI. So, for instance, a person's behavior can be explained by postulating traits of her character: if you ask me, "Why did John yell at me?," I could answer "Because he has an irascible character and you provoked him." In doing so, I postulate a new entity, a character trait, that accounts for an MI behavior. These sorts of postulation typically introduce other layers of correlational explanations; so, in the case in point, the postulation of irascibility 
explains John's yelling because irascible characters regularly produce violent reactions if provoked. The explanation may be considered a good one because that character trait supports different correlations. While the postulation of a virtus dormitiva only allows us to predict that opium makes one sleep in every situation, the postulation of character traits allows to predict different behaviors in different contexts: e.g. irascible people yell at friends if provoked, are assertive if contradicted, smash things if they do not work as they expect, and so on. However, since the only explanation that this kind of postulation provides is of the correlational sort, it squarely belongs to MI. This is the reason why we call them spurious.

13 SI postulated entities, instead, are explanatory in a genuine sense. They are theoretically autonomous from MI objects in that they are subject to a different kind of normativity: they obey only statistical laws rather than dispositional regularities. Consequently, they do not go proxy for dispositions of MI objects. In other words, SI postulated entities are autonomous because they are not constituted according to the category of person, which is the fundamental category of MI. The determination of a new normative space is integral to the genuine postulation of the SI entities. When an entity is postulated, the normative space within which the postulated entity is defined is concomitantly established and constituted. "Concomitantly" here should be taken in a logical rather than temporal sense: postulated entities and the normative spaces to which they belong are intrinsically and essentially related. The explanatory power of those entities depends on their belonging to a normative space constituted by specific rules and principles. A normative space allows us to draw inferences that articulate the content of the sentences where reference to postulated entities occurs, thus providing a pattern of explanation which, in turn, ultimately accounts for the behavior of MI objects. The intrinsic and essential relation between postulated entities and their underlying normative space is a pivotal feature of our account of postulation.

It is important to stress that the distinction between spurious and genuine postulation does not correspond to the distinction between the postulation of ostensible and real entities. It might be argued that the difference between the two should come down to the fact that genuinely postulated entities are real if the corresponding theory is true, while spuriously postulated entities are merely fictional. This reading of the distinction is based on an assumption that we reject, for a twofold reason. That assumption is the idea that the qualifications 'genuine' and 'spurious' have to do with truth. Firstly, it is not correct to say that spuriously postulated entities are not real: actually, they are real within MI, if the corresponding theory is true, since they are adequately constructed according to the fundamental category of that normative space, namely the category of person. Secondly, the postulation of entities may be genuine even in the case in which the corresponding theory is false. Such a distinction is therefore preliminary and independent from the empirical investigation about the reality of postulated entities. To say that entities are genuinely postulated is noncommittal with respect to the adoption of a realist or an instrumentalist stance towards them.

15 It is important to see clearly why the normative space of SI genuinely postulated entities cannot be integrated within the normative space of MI. As we have already pointed out, we rely on Sellars' insight that the basic ontological category of MI can be characterized in terms of the concept of person, and the normativity of the correlations that articulate its explanatory framework in terms of the powers and dispositions of person-like substances. So consider, for instance, the MI statement "Light bulbs light up when 
crossed by electric current." This commonsensical principle expresses a correlation between some manifest properties of certain substances, where the correlation essentially depends on their form. So, in our example, a light bulb is a substance that has the potentiality to light up when acted upon by another substance, the electric current (a spuriously postulated entity). SI statements, instead, do not primarily ascribe properties to substances. In a general sense, to define a scientific theory is to define a collection of laws. Such a definition is possible only within a normative space articulated in terms of inferential relations. ${ }^{8}$ Obviously, a collection of laws may or may not have models. The objects of a scientific theory are the entities in the domain of the structures that satisfy it. Satisfaction here is, of course, a semantic notion rather than an empirical one. In this sense, the objects of a scientific theory essentially belong to the normative space of the theory itself and do not have any essence independent of it. Accordingly, they just cannot be integrated in the normative space of MI. The question whether or not the new postulated entities exist is of course an ontological one. Now, both the manifest and the scientific image are intended to accommodate complete representations of the world in the sense that, ideally, they should both provide the resources to give an explanation of the same events. Therefore, since they have different ontologies, the two images are incompatible. It is important to notice, however, that while such an incompatibility more strikingly comes to light as a clash between different sorts of ontologies when models for manifest and scientific theories are considered, nonetheless it lies primarily in the structure of the manifest and the scientific normative space. In our view, ontological issues are traced back to normative ones.

Up to this point, we have taken into account the logical structure of postulation from the point of view of the inferences that the act of postulation allows us to make. In doing so, we have been concerned with the language of SI and MI, since inferences are relations between sentences or propositions. From a pragmatist point of view, however, it is not enough to investigate the distinctive features of the final result or outcome of a certain act; it is also necessary to state the problems that such act is expected to tackle. In this sense, it is important not only to understand what postulation consists of and what its consequences are, but also why one should avail oneself of it. As may be expected, the answer is that postulation is required when correlational explanation is not enough to provide a satisfactory account of why something is the case. In Peircean terms, postulation is a necessity of inquiry when doubt cannot be appeased by purely correlational means. The source of doubt can take different forms. One might bump into a contradictory situation, just like when the result of an experiment contradicts a law. Or one might simply wonder how something really works, as when the reasons why a certain law is valid are investigated. Often, it is a bit of both. Consider again, for instance, the Boyle-Charles law. It was originally proved by Boyle in the 17th century on purely empirical evidence by correlating variations of volume and pressure in gases at a fixed temperature. The law, in effect, is valid only for ideal gases, but the fact that the behavior of real gases diverts from it can be appreciated only when the law is tested at particularly low temperature or high pressure. Such results were not originally available to Boyle and yet, once they became apparent, they obviously required a different explanation. Such an explanation was in fact provided in 1738 by Bernoulli, who first defined the basis of the kinetic theory of gases by postulating that gases are composed by imperceptible particles, whose motion determines their macroscopic properties and behavior. Quite interestingly, Bernoulli's approach was completely adopted only a century later, when physicists gained clarity about the relation between heat and kinetic energy. 


\section{Ways of Practicing the World}

\subsection{An Elucidation of Pragmatic Content} characteristically bound by norms. The correctness of what we do or not do is subject to the independent valuation of our social peers. As social practitioners, we are provided with normative statuses that specify what we are allowed to do and what we are committed to doing. This normative social environment that we inhabit is responsible for the sort of cultural learning that, together with the biological endowment that we inherited through evolution, accounts for our multifarious abilities to cope with the world (see also Dewey 1938; Margolis 2016, in particular Chapter 1). Concept application is one such ability that allows us to track things in the world, acquire and modify information about them, predict their behavior, and so on. The practices involving concept applications are governed by social norms as much as any other. In this sense, what distinguishes my ability to make observational judgments about colors from a trained parrot's reliable disposition to croak "that's red" when confronted with red surfaces is that I can handle the normative premises and consequences of my judgment: e.g. that I am not entitled to apply the concept red in a dark room feebly illuminated by a candle as I apply it in daylight, that I am committed to apply the concept colored to anything to which I have applied the concept red, and so on. As it is easy to see, this is but a pragmatist presentation of Wilfrid Sellars' seminal notion of the space of reasons, "of justifying and being able to justify what one says" (Sellars 1956: 169). In this context, justification is not a merely linguistic affair, but is to be construed as the vindication of the entitlement to the commitments that one has endorsed.

It is also easy to see, at this point, how the normative articulation of the practices that involve concept application determines conceptual contents. In fact, the content of a specific concept can be functionally defined in terms of the normative relations that are practically established between its applications and the applications of other concepts. When the expressive resources of logical vocabularies are available, these normative 
relations can also be made explicit in terms of inferential relations between sentences. Hence, an inferentialist semantic analysis can be introduced: the content of a sentence is determined according to its inferential role as the couple made of the set of the sets of premises from which it can be inferred and the set of the sets of consequences that can be inferred from it together with other sets of sentences. ${ }^{10}$

This should be enough to envision how an answer can be given to the first of the questions considered above. In fact, conceptual contents can be functionally determined in terms of the web of normative relations that govern them. A semantic definition of this sort, however, is not representational. ${ }^{11}$ It might still not be clear, then, how the judgments in which conceptual contents thus defined are applied can be about things in the world; it is therefore worth saying a few words about this point. To begin with, the reason why this seems to be an easy hurdle to clear for a representationalist semantics is that such a semantics is based on the view that to be meaningful is just to have representational content. However, representationalist semantics obtains this result at the cost of taking for granted the notion of representation. In other words, it guarantees that expressions have representational contents, but, in fact, it does not really explain what it is for an expression to represent something in the world. In our approach, conceptual contents are defined in terms of the normative relations that are established in a practice. In this sense it is a decisively anti-representationalist approach. ${ }^{12}$

There is, however, another sense in which our approach is entirely compatible with the idea that sentences have representational content. To begin with, we obviously agree that collections of laws expressed in the language of a theory may have models, and that models can play the role of semantic representations for the conceptual contents defined by the laws. We merely contend that it is normative practices rather than the existence of models that make a theory meaningful. We also do not deny that the objects in the domain of the models of a theory might be said to really exist, on the basis of a Quinean metaontology. We simply do not think that the ontology of the language of theories is the proper framework in which to approach the problem of the objectivity of a conceptual repertoire developed within a normative space. Instead, we believe that normative spaces and the real world make contact through 'thick' normative practices. By the expression 'make contact' we refer to the fact that the language of theories spins in the void - it cannot grasp the world conceptually - if it is not intrinsically related to, and supplemented by, normative practices in which we are directly confronted with the world. Such direct confrontation typically takes the form of physical manipulation, instrumental interference, and so on. We label 'thick' those practices that essentially involve a concrete, manipulative transaction with things in the world. ${ }^{13}$ It is easy to realize how this is the case for MI practices. Accordingly, we proceed to show that the same holds for SI practices.

\subsection{A Grammar of Scientific Practices}

23 Hasok Chang has done a large amount of preliminary work in the way of highlighting what he calls the grammar of scientific practice. In a series of articles devoted to assessing the recent practical turn in philosophy of science, Chang has claimed that the traditional practicalist approach aimed at singling out the various, different elements i.e. the various, different syntactic operations, as well as the laboratory's material equipment - that enter into experimental practice should be integrated with an analysis 
of the ways in which they concretely combine and interact with each other (Chang 2011, 2014). We cannot rest satisfied with a taxonomy of experimental practice put in linguistic terms, he argues; what we need to know is the life of those elements. The structure of such life is what Chang refers to with the label 'grammar of practice,' which consists in the possible ways - 'possible' here is not intended in a strictly logical sense, but rather in a material and functional sense, like when one lists the possible ways of building a house or cultivating a cornfield - in which a complex group of epistemic activities may consistently work. We take the idea of groups of epistemic activities to be synonymous with our notion of SI practices.

Chang's goal is similar to ours; he also aims to avoid the disconnection between science and its practice. The kind of disconnection that Chang has in mind is the by-product of the habit of focusing on the results of scientific investigation rather than on the processes that yield them, with that habit representing the standard view among analytic philosophers of science. According to this view, scientific theories are bodies of propositions, and philosophical problems about them can be assessed by investigating the logical relationships between those propositions. Therefore, the role of SI practices in bringing about SI languages is almost completely neglected: as a consequence of that move, the concrete life of science is substituted with a logical or mathematical analysis of the relations holding between the elements of the theories - whether conceived of in terms of linguistic propositions or set-theoretic structures.

It is very interesting that, as a possible way of defusing the threat of disconnection, Chang suggests shifting the attention from nouns to verbs. So, for instance, take 'representation' and think of it as 'representing,' take 'causality' and think of it as 'causing,' and so on. In doing so, the active character of scientific knowledge is brought under the spotlight. While 'representation' seems to entail a sort of simple, direct relation to the thing represented, 'representing' implies a more complex network of relations, centered on the role and function of the epistemic agent engaged in the activity of representing a state of affairs for a specific purpose in a specific context. This move sounds very Deweyan in spirit. It was Dewey who firstly suggested to treat adjectives and nouns as adverbs: let's not talk of intelligence or rationality, but rather of an intelligently conducted activity; let's not talk of a true belief, but rather of a truly reconstructed situation. ${ }^{14}$ Thanks to that shift of perspective, the issue can be framed in a radically different manner, and new questions emerge and wait to be answered. Those questions concern the epistemic activity of the knowing subject: "who is doing what, why, how, and in what context?"

Nonetheless, focusing on verbs (or adverbs) rather than on nouns (or adjectives) is not enough to grasp the concrete reality of the practices that comprise scientific activity: the point is that, by talking of representing instead of representation, we remain on a purely linguistic level. At best, we succeed in providing an extremely ethereal account of the epistemic activities involved in SI practices, grounded on the triviality that to speak is to act. An account of this kind could be labeled 'pragmatist' only in a minimal and wholly uninteresting sense. Consequently, a further step should be taken, which brings to the fore the concrete set of epistemic activities associated with a certain verb.

We are brought back to the idea of the thickness of SI practices. In a functionalist and pragmatist fashion, Chang states that "all scientific work, including pure theorizing, consists of actions - physical, mental, and 'paper-and-pencil' operations" (Chang 2011: 208). This is a genuinely pragmatist move in that it draws attention on the material conditions of possibility of SI sentences. The production of SI sentences is part of the SI 
practices in which the agent is engaged. On the one hand, SI sentences are proffered at a certain moment, for a specific purpose. Usually, they are proffered with an eye to the consequences that can be brought about by their utterances - i.e. to record an event, to describe a particular phenomenon, to establish an experimental setting, and so on. On the other hand, the utterances of SI sentences are checked and controlled by the other operations with which they continuously interact. It is for this very reason that a taxonomy of the elements of the scientific practice is not enough to understand what scientific activity is. Without a normative framework in which operations are performed and interact with each other, providing a list of the elements that compose a scientific practice is not explanatory at all. It is like listing the elements in a toolbox without providing any information about their function and forms of employment; such knowledge would be blatantly insufficient. Coherent sets of epistemic activities are the overall normative context in which operations are performed and can exert their functions. For a set of epistemic activities to be coherent, it has to be directed towards a certain end - paradigmatically, the acquisition of a certain bit of knowledge or, to put it in pragmatist terms, the solution of a specific problematic situation - in accordance with some set of discernible rules. ${ }^{15}$ As Chang remarks, "[b]ecause activities are rule-bound systems of actions, they are inherently normative in the sense that the actions within an activity are continually evaluated in terms of their conformity to the rules" (Chang 2011: 209).

We agree with Chang on all these points, but we are ready to take a step further. The point we would like to stress is that the insistence on the complex nature of SI practices paves the way for a globally consistent anti-representationalist account of the semantic content of scientific concepts. The difference between our two approaches can be highlighted by an example. Speaking of how the nature of a definition should be conceived once we take a practical turn, Chang says:

[I]nstead of thinking about the nature of a definition, we can consider what one has to do in defining a scientific term: formulate formal conditions, construct physical instruments and procedures for measurement, round people up on a committee to monitor the agreed uses of the concept, and devise methods to punish people who do not adhere to the agreed uses. (Chang 2011: 208)

In our view, this set of epistemic activities establishes the normative rules for the use of an SI concept. We are concerned with the conditions of possibility of SI definitions, and we trace them back to the SI practices that are necessary to master the use of the concept. In more general terms, we hold that the complex set of syntactic operations, practical skills needed to carry out scientific experiments in a laboratory context, and 'institutional capabilities' that make it possible for a researcher to be part of a community (scientific, technological and democratic) is all that is necessary to use an SI concept. So, for instance, to fully master the SI concept of atom one must (1) handle in a competent manner the mathematical and syntactical tools necessary to formulate the best atomic theory available; (2) master the laboratory equipment required to detect or modify the behavior of atoms; (3) be aware of the rules governing the different communities to which she belongs as a scientist, as a citizen, as a possible patent holder, etc. ${ }^{16}$

A qualification here is needed: we have said that to fully master an SI concept one must display three different kinds of capacities. It is important to properly understand what is conveyed by the adverb 'fully.' 'Fully' here does not mean completely: that would amount to a too restrictive clause. It would entail that, in order to use in a normatively adequate way the concept of atom (and the corresponding linguistic expression), one should 
possess perfectly developed capacities in many different fields. If that were the case, nobody could be said to master the SI concept of atom, which is an undesired skeptical result. What we have in mind here is something like Collins and Evans' distinction between contributory and interactional experts. In their book Rethinking Expertise, Collins and Evans stress the difference between contributory expertise, which is required to do an activity with competence, and interactional expertise, which is "the ability to master the language of a specialist domain in the absence of practical competence" (Collins \& Evans 2007: 14). Similarly, we argue that it is possible to master some parts of the SI language without fully mastering them, that is, without mastering the practices that establish the normative conditions for the use of SI concepts. The 'interactional' use of SI language, so to say, is therefore parasitic to the 'contributory' use consisting in the mastery of SI practices.

Before moving on to the next section, where a comprehensive account of the relationships between MI and SI is provided, we would like to address an issue which has been left untouched until now. In section 2 we have acknowledged the introduction of postulated entities for explanatory reasons as the distinctive feature of the SI normative space. In that context, we have argued that the distinction between MI and SI normative spaces consists precisely of the fact that SI language allows and supports genuine postulational activity, a postulational activity being genuine when the entities it introduces are not conceived in terms of the categories of MI. What remains to be done is therefore to highlight how such a postulational activity shows up at the level of practices, thus marking a difference between SI and MI practices.

We should be careful, however, not to overemphasize the elements of discontinuity. Because of the very structure of a practice, the clear-cut differences that can be easily detected at the linguistic level are inevitably blurred at the level of practices. In the last analysis, every practice, no matter how complicated and abstract it might be, comes down to a manipulation - both physical and symbolic - of natural objects, events and worldly states of affairs. SI practices are particularly complex because they are made of extremely refined epistemic activities, such as testing, constructing models, measuring, calculating, writing, classifying, and so on; however, they are not essentially different from MI practices. Ultimately, SI practices are human activities which stem from the technological, formal and normative refinement of the artifactual, linguistic and social resources of common sense. The difference between MI and SI practices is therefore, in a sense, a matter of degree: SI practices can be (and indeed have been) elaborated from MI practices. Clearly, the recognition of a certain continuity does not imply the thesis that there is no difference between them; there is, as everybody who ever took part to the activity of a scientific laboratory knows perfectly well! We do not want to deny the differences between MI and SI practices; our point is simply that the clear discontinuity that exists between SI and MI languages cannot be found among MI and SI practices.

With this in mind, we go back to the issue concerning the form taken by postulational activity in SI practices. Our solution is inspired by Hacking's experimental conception of effects or phenomena. In Representing and Intervening Hacking remarks that phenomena should be conceived of as regularities which are consequences of the laws of nature formulated in our theories. Contrary to the philosophical sense of the word 'phenomenon,' Hacking does not use it to refer to something private, but rather to "something public, regular, possibly law-like, but perhaps exceptional" (Hacking 1983: 222). When a phenomenon is particularly interesting and instructive, scientists call it an 
effect. Consequently, a phenomenon is the result of an experimental SI activity that alters more or less dramatically the course of nature, thus creating a regularity that would be otherwise inaccessible through MI practices.

What is relevant to note is that, according to Hacking, effects do not exist "outside of certain kinds of apparatus." "In nature," he argues, "there is just complexity, which we are remarkably able to analyse. We do so by distinguishing, in the mind, numerous different laws. We also do so, by presenting, in the laboratory, pure, isolated, phenomena" (Hacking 1983: 226). What 'exists' (holds) independently of our experimental SI practices are the laws of nature; on the contrary, effects and phenomena do not exist until an experimenter discovers how to disentangle a particular arrangement which exemplifies - rather than merely instantiates ${ }^{17}$ - a particular effect or phenomenon by purifying it from other intervening and interfering causes.

Following up on Hacking's insight, we argue that an interesting way to think of the postulational activity at the level of SI practices is in terms of Cartwright's 'nomological machines, ${ }^{18}$ i.e., in terms of the construction of experimental settings which make it possible to produce effects or phenomena in a laboratory context. Such a comparison is warranted by the fact that the construction of an experimental setting is a kind of activity which is structurally similar to the act of postulation, since in the former a modification of some natural conditions is produced in order to account for correlations that would be left otherwise unexplained. In both cases, the search for correlations is supplemented by the introduction of some factors that dramatically enhance the explanatory power of scientific activity - nomological machines at the level of SI practices, and postulated entities at the level of SI languages. There are two more similarities that are worthy of note. First of all, the construction of an experimental setting cannot be severed from the theory that allows the particular, specific regularity exemplified by that arrangement. Consequently, it is reasonable to argue that they share the same structural complexity. Secondly, both the postulated entities and the nomological machines do not exist or hold outside of a well-defined context, respectively the SI language of the theory and the laboratory SI practices. ${ }^{19}$

\section{Meaning-Use Analysis}

In sections 2 and 3 we have analyzed the languages and the practices of science and common sense as they come into view in MI and SI. We have also discussed the pragmatic significance of the conceptual contents that are expressed in these languages and we have maintained that practices are fundamental to their determination. In fact, the way in which normative spaces have been presented prefigures an analysis of languages and practices as deeply intertwined. In this section we will try to offer a sharper picture of the relations between the various components of MI and SI. In order to do that, we will exploit the expressive resources of Brandom's so called "meaning-use analysis," which is essentially a collection of theoretical tools designed to provide a pragmatist analysis of languages and their semantic contents (Brandom 2008).

Our purpose is to argue that in a normative inferentialist approach, like the one we have endorsed here, the traditional ontological conflict between science and common sense is shifted from the level of the semantic analysis of languages to the level of the analysis of normative practices. One of the most efficacious illustrations of such a conflict is Eddington's example of the two tables. Eddington suggested that if the ontological 
commitments of science and common sense are taken at face value, then it must be acknowledged that for every common sense object there exists a scientific duplicate that is distinct from it. Consider a table, for instance. On the one hand there is the table of common sense, an MI object with phenomenological properties like extension, color, shape, impenetrability, weight, etc. On the other hand there is the table of science, a mostly empty space in which many electric charges move around. Whereas the MI table is a substance with properties, the SI table is hardly something at all. There is an unquestionable ontological distinction between the two tables. The problem arises when the representational content of our assertions about tables is taken into account: when we talk about tables, do we refer to the MI or the SI one? Since we cannot refer to both, in establishing whether our assertions are true or false should we adopt the ontology of common sense or the ontology of science? This is what the problem is usually taken to be.

The most common and accredited interpretation of Eddington's example admits that the references to the two tables are in effect semantically incompatible, because the objects they refer to are ontologically incompatible with each other. Once this is acknowledged, however, one seems to be forced to choose one table or the other. So, on the one hand, scientific realists acknowledge the existence of the SI table only and lessen the ontology of common sense as phenomenalism. On the other hand, those who stick to the MI table like, for instance, phenomenologists who vindicate the primacy of the life-world endorse an instrumentalist approach to scientific theories, according to which the latter are nothing but tools useful to cope with the world, which can be legitimately employed only with the proviso that they allow us to save the phenomena. Although Eddington's picture describes a sound ontological dilemma, we believe nonetheless that the conflict between science and common sense cannot be settled by picking one of its horns. We suggest, instead, that a better understanding of the relationship between these two frameworks can be achieved by opting out of the ontological plan altogether. In order to do that, we need to investigate more in depth how the MI and SI normative spaces are interrelated.

As remarked above, the two main components that characterize a normative space are a language and a practice. We thus distinguish, on the one side, the language and the practice of MI $\left(L_{M}\right.$ and $\left.P_{M}\right)$ and, on the other side, the language and the practice of SI $\left(L_{S}\right.$ and $\left.P_{S}\right)$. Here, $L_{M}$ is the language in which we talk about tables and their colors, the language in which we formulate the Boyle-Charles law for gases, and so on. On the other hand, the practice $P_{M}$ is the ordinary practice of dealing with these substances and their properties, in the peculiar way that characterizes us as rational human beings and that consists in having the responsibility to justify what we do. Similarly, $L_{S}$ is the language of science in which we talk about atoms and report gravitational waves, while $P_{S}$ is the thick scientific practice that we have described in the previous section.

The first kinds of relation that we want to single out connect languages and practices inside the same normative space. We have argued that practices are fundamental to the determination of the conceptual contents expressed in MI and SI languages. As a consequence, in order to be treated as being able to meaningfully deploy the vocabularies of $L_{M}$ and $L_{S}$, one must already be able to engage in the correspondent practices $P_{M}$ and $P_{S}$. This is a relation that Brandom calls "practice-vocabulary-sufficiency" (PV-Sufficiency), because it holds when the condition of engaging in a certain practice is sufficient for the meaningful deployment of a certain language. In this sense we have both that $P_{M}$ is PVSufficient with respect to $L_{M}$ and that $P_{S}$ is PV-Sufficient with respect to $L_{S}$. 
41 Another relation that Brandom considers is the inverse "vocabulary-practice-sufficiency" (VP-Sufficiency), which holds between a certain language and a certain practice when the language contains the expressive resources to specify what one must be able to do in order to engage in the practice. Since both MI and SI are ideal types that contain the resources to accommodate complete representations of the world, the languages $L_{M}$ and $L$ ${ }_{s}$ in effect must have enough expressive power to specify what it is to engage respectively in $P_{M}$ and $P_{S}$. Thus $L_{M}$ is VP-Sufficient with respect to $P_{M}$ and $L_{S}$ is VP-Sufficient with respect to $P_{S}$.

Let's pause to take stock. So far we have defined VP-Sufficiency, an expressive relation between languages and practices, and PV-Sufficiency, a semantically grounding relation between practices and languages. These few instruments already allow us to ask an interesting question: What if a language $L^{\prime}$ is VP-Sufficient with respect to a practice $P$ that in turn is PV-sufficient with respect to a language $L "$ "? This question invites to reason about a scenario in which a language $L^{\prime}$ is expressive enough to specify a practice $P$, the engaging in which is sufficient for the meaningful deployment of another language $L^{\prime \prime}$. In this scenario a composite relation between the languages $L^{\prime}$ and $L^{\prime \prime}$ can be envisaged, one that is mediated by the practice $P$. Here, in a sense, $L^{\prime}$ allows to express the contents of $L^{\prime \prime}$, to the extent that it allows to specify the practice that establishes them. And yet, $L^{\prime}$ is not a semantic metavocabulary for $L^{\prime \prime}$, because it does not talk about the expressions of $L^{\prime \prime}$. Thus Brandom calls $L^{\prime}$ a "pragmatic metavocabulary" for $L^{\prime \prime}$. Notice that it is not necessary for $L^{\prime}$ and $L^{\prime \prime}$ to be different languages. In fact, it is easy to verify that both $L_{M}$ and $L_{S}$ are pragmatic metavocabularies of themselves. This should not be surprising: since the languages of MI and SI in our analysis must be expressively complete, they contain the resources to specify the practices that ground their contents. ${ }^{20}$

We can then proceed to consider whether there are relations holding between the different normative spaces of SI and MI. If we focus just on $L_{M}$ and $L_{S}$, however, the picture is not really encouraging. Eddington's example suggests that the languages of the two images are on the whole semantically autonomous. Surely, there is no semantic reduction between them. So, for instance, reference to tables can be reduced to reference to electric charges with kinetic energy only at the price of obliterating the whole MI as a normative space.

The overview however changes substantially when practices are taken into account. For it is easy to see that the language of SI has the expressive power to specify the practices of MI. Consider the following example. To be able to apply the MI concept of a light bulb is, among other things, to acknowledge that flipping the light switch will turn the light bulb on. The expression 'flipping the light switch will turn the light bulb on' is a norm expressed in $L_{M}$ that governs the practice of application of the concept of light bulb. Such a norm, however, can also be specified in $L_{S}$. In order to do that, the lighting of the light bulb is to be represented in SI. Physicists explain that the electrons that are accelerated by the electric field created by flipping the switch collide with the atoms of the filament in the light bulb. The electrons of these excited atoms transition into higher energy levels. Quantum electrodynamics says that atoms may spontaneously transition back to their ground state: when they do so, electromagnetic radiation is emitted in the form of photons. The wavelength of the emitted photons depends on the distance between the energy levels of the atomic transition. The material and the mass of the filament inside a light bulb are such that the probability of photons being emitted in the visible 
wavelengths is high enough for the light bulb to glow. One can then specify what it is to be able to apply the MI concept of a light bulb in $L_{s}$ by saying that it involves, among other things, acknowledging that, when the light switch is flipped, an electric field excites the atoms of the filament in the light bulb that spontaneously emit photons in visible wavelengths when they jump back to their ground state. Notice that although it is specified in $L_{S}$, the practice of turning on light bulbs is not a scientific one. This example illustrates that it is possible to say in $L_{S}$ what one does when one engages in $P_{M}$. In this sense, then, $L_{S}$ can be deployed as a pragmatic metavocabulary for $L_{M}$. So, while it is not possible to reduce the contents of the language of MI to the contents of the language of SI, it is still possible to use $L_{S}$ to talk about the practices that establish the norms that determine the contents of $L_{M}$.

The pragmatic point of view also allows to see that there are interesting relations between $P_{M}$ and $P_{S}$ too. As far as the analysis of the conflict between MI and SI is concerned, the most interesting relation that we want to single out is the one from $P_{M}$ to $P$ ${ }_{s}$. As was explained in the previous section, we see $P_{S}$ as characterized by the complex collection of epistemic activities that allow for the formulation and testing of scientific theories. The normative space of SI only obtains when these activities are up and running in scientific practices. However, the development of all the formal, material and social resources required to start and sustain them is a complex process. Such a process consists, among other things, in defining languages and theories with new expressive power, in constructing new instruments and equipment and learning how to use them, in establishing new social groups and normative statuses. It is important to realize that the development of all these heavily relies on the resources that are available in $P_{M}$. Let us start with the most obvious case: although experimental equipment enables SI abilities, they are in effect macroscopic objects, so designing, constructing and operating them requires a whole series of MI abilities. Similarly, the ability to ascribe SI normative statuses to scientific communities and institutions requires the ability to ascribe MI normative statuses to people. The case of the development of SI formal resources is slightly more ponderous. We will present it by means of an example: consider the notion of integral, which is employed to express wave functions in the definition of particles like electrons. Integrals do not belong to MI. By contrast they are part of the expressive resources required to engage in SI practices. If we look at the history of the notion, however, we clearly see how the development of the ability to deploy the concept of integral consists in a progressive elaboration of other abilities that were previously available in MI. Leibniz, for instance, thought of integration as an infinite sum of ordinates with infinitesimal abscissas. ${ }^{21}$

Admittedly, these remarks are only of an introductory character, but they are enough to outline the relation of elaboration between the practices of MI and SI that we want to focus on. This relation holds between $P_{M}$ and $P_{S}$ because the resources required by the epistemic activities that characterize $P_{S}$ can be elaborated from the resources that are already available in $P_{M}$. Although it is a very peculiar relation specific to $P_{M}$ and $P_{S}$, it is clearly a species of the more general genus that Brandom calls PP-Sufficiency relations. These hold, he explains, "when having acquired one set of abilities means one can already do everything one needs to do, in principle, to be able to do something else" (Brandom 2008: 26). For want of a better word, we will simply refer to the sort of elaboration that we have described of $P_{S}$ from $P_{M}$ as PP-Sufficiency. In this case $P_{M}$ is PP-sufficient for $P_{S}$. 
In order to wrap this all up, all the relations that have been described here are depicted in Figure 1.

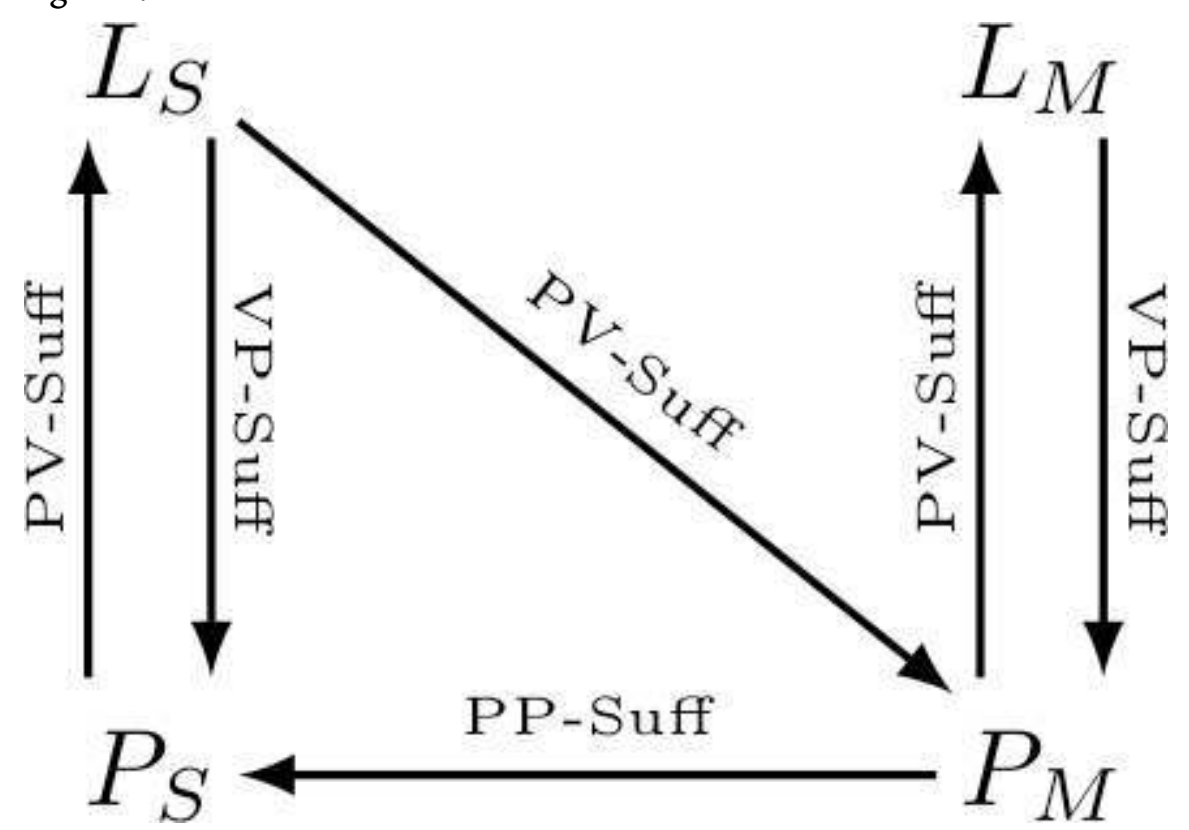

Figure 1: meaning-use analysis

If we now look at the whole picture drawn by this analysis, we can see that is has several points of strength. First of all, it acknowledges that the evident ontological clash between $\mathrm{SI}$ and MI is a sound one. The conflict follows from the fact that the semantic analyses of $L$ ${ }_{s}$ and $L_{M}$ are incompatible in the strong sense that the models that make $L_{S}$ sentences true are not isomorphic to the models that make $L_{M}$ sentences true. In a Quinean metaontology, this means that $L_{S}$ and $L_{M}$ talk about different things: if $L_{S}$ sentences are true then wave functions exist, if $L_{M}$ sentences are true then instead tables exist. And since SI and MI are ideally complete representations, wave functions and tables cannot exist both. So, either $L_{S}$ or $L_{M}$ sentences must not be really true. In our analysis, however, while model-theoretic semantics is accepted as an essential expressive resource, it does not play a foundational role with respect to the meaning of the expressions of a language. In particular, the conceptual contents of $L_{S}$ and $L_{M}$ are construed as determined by the inferential articulation of the norms established in the respective practices $P_{S}$ and $P_{M}$.

Hence, since the practices of science and common sense are clearly intertwined, our analysis also allows one to account for the continuity between the two frameworks. In particular, it makes room for an explanation of the process of development and enrichment of common sense. Here we have sketched some of the lines along which it is possible to investigate how a common sense practice like $P_{M}$ can be elaborated into a scientific one like $P_{S}$. At the same time, however, we have not blurred the distinction between the practices of science and common sense. While MI can make use of methods that are usually taken to be characteristic of scientific activity, like idealization, induction, abduction and statistical inference, SI is still distinguished, both on the level of practices and on the level of languages, by its distinctive postulational activity. 


\section{Conclusions}

50 between science and common sense. In particular, we have been concerned with the
structure that can be given to a comparison between the two frameworks. Therefore, we have focused on a specific area in which they overlap, an area that we have delimited in terms of the notions of SI and MI and that we believe may paradigmatically highlight the shape of their connection. As we have defined them, SI and MI are normative spaces that are differently characterized by practically established norms. These norms functionally determine the conceptual contents which are applied to give linguistic representations of things in the world. Traditionally, the comparison between science and common sense has been drawn at the level of these linguistic representations. And since these linguistic representations are semantically incompatible, the two frameworks have been regarded as mutually exclusive. In our approach, instead, we suggest investigating the conflict between science and common sense at the level of the practices. Thus, we give an analysis of SI and MI practices that accounts for the distinction between the postulational character of the former and the merely correlational norms of the latter. At the same time, we show how SI practices can be elaborated from MI ones and are therefore continuous with them. By focusing on practices, our approach also allows us to shed a different light on the relation between the languages of science and common sense. In fact, we note that SI language is a pragmatic metavocabulary for MI language, in the sense that it is possible to specify in the former what one must be able to do in order to engage in the practice that semantically grounds the latter.

of course, all this only scratches the surface of the relationship between the practices of science and common sense, and we think that a lot of promising work is still to be done in this area. ${ }^{22}$ We also believe that the very possibility of reorienting the focus of such investigation away from ontological conflicts and towards their practical roots is a relevant contribution of our pragmatist approach. Among the values of such an approach we also count the fact that it keeps together different conflicting intuitions that all seem to be valid. In particular it allows to acknowledge the incompatibility between conceptual repertoires of science and common sense, but it also allows us to treat such incompatibility as non-malicious, one that can be resolved by taking into account the continuity of practices. Accordingly, it suggests that there is no need to look for an alleged fixed group of theses which are essential to MI: common sense evolves and is permeable to the results of scientific investigations. Finally, since it moves from the assumption of a continuity between science and common sense, our account may provide some elements to explain why in the history of science phenomenological laws have proved to be quite independent from the scientific explanations that were given for them. The point is that phenomenological laws belong to common sense, being correlations between manifest properties of manifest objects. That suggestion paves the way for a pragmatist analysis of the relations between operational practices - such as measurement - and SI languages, much in the spirit of Chang's recent reappraisal of operationalism (Chang 2004, 2012, 2017). 


\section{BIBLIOGRAPHY}

BRANDOM Robert, (1994), Making It Explicit: Reasoning, Representing, and Discursive Commitment, Cambridge, MA, Harvard University Press.

BRANDOM Robert, (2000), Articulating Reasons, Cambridge, MA, Harvard University Press.

BRANDOM Robert, (2008), Between Saying and Doing. Towards Analytic Pragmatism, Oxford, Oxford University Press.

BRANDOM Robert, (2011), Perspectives on Pragmatism: Classical, Recent and Contemporary, Cambridge, MA, Harvard University Press.

CARNAP Rudolph, (1934), Logische Syntax der Sprache, Vienna, Springer. En. tr. by Amethe Smeaton, The Logical Syntax of Language, London, Kegan, Paul, Trench Teubner \& Cie, 1937, Paterson, NJ, Littlefield, Adams \& Co., 1959, Chicago, Open Court, 2002.

CARTWRIGHT Nancy, (1999), The Dappled World. A Study of the Boundaries of Science, Cambridge, Cambridge University Press.

CHANG Hasok, (2004), Inventing Temperature: Measurement and Scientific Progress, Oxford, Oxford University Press.

CHANG Hasok, (2011), "The Philosophical Grammar of Science," International Studies in the Philosophy of Science, 25 (3), 205-221.

CHANG Hasok, (2012), Is Water $\mathrm{H}_{2} \mathrm{O}$ ?: Evidence, Realism and Pluralism, Dordrecht, Springer. CHANG Hasok, (2014), "Epistemic Activities and Systems of Practice: Units of Analysis in Philosophy of Science After the Practice Turn," in Léna Soler, Sjoerd Zwart, Michael Lynch, \& Vincent Israel-Jost (eds.), Science After the Practice Turn in the Philosophy, History, and Social Studies of Science, New York, Routledge, 67-79.

CHANG Hasok, (2017), “Operationalism: Old Lessons and New Challenges,” in Nicola Mößner \& Alfred Nordmann (eds.), Reasoning in Measurement, New York, Routledge, 25-38.

COLLINS Harry \& Robert EVANS, (2007), Rethinking Expertise, Chicago, University of Chicago Press. DEWEY John, (1906), "Experience and Objective Idealism," in The Middle Works of John Dewey, Volume 3, 1899-1924: 1903-1906, edited by Jo Ann Boydston, Carbondale, IL, Southern Illinois University Press, 128-44.

DEWEY John, (1920), Reconstruction in Philosophy, in The Middle Works of John Dewey, Volume 12, 1899-1924: 1920, edited by Jo Ann Boydston, Carbondale, IL, Southern Illinois University Press, 77-202.

DEWEY John, (1938), Logic: The Theory of Inquiry, in The Later Works of John Dewey, Volume 12, 1925-1953: 1938, edited by Jo Ann Boydston, Carbondale, IL, Southern Illinois University Press. DUMmETt Michael, (1977), Elements of Intuitionism, Oxford, Clarendon Press. Dummetт Michael, (1991), The Logical Basis of Metaphysics, London, Duckworth. FRANCEZ Nissim, (2015), Proof-theoretic Semantics, London, College Publications. 
FREGE Gottlob, (1879), Begriffsschrift: Eine Der Arithmetische Nachgebildete Formelsprache des Reinen Denkens, Halle, Nebert. En. tr. by Stefan Bauer-Mengelberg as Begriffsschrift, A Formula Language, Modeled Upon That of Arithmetic, for Pure Thought, in Jean van Heijenoort (ed.), From Frege to Godel, Cambridge, MA, Harvard University Press, 1967.

GENTZEN Gerhard, (1934-5), “Untersuchungen fiber das logische Schliessen," Mathematische Zeitschrift 39, 176-210, 405-31. En. tr. by Manfred E. Szabo as "Investigation into Logical Deduction," in M. E. Szabo (ed.), The Collected Papers of Gerhard Gentzen, Amsterdam, New Holland, 1969.

HACKING Ian, (1983), Representing and Intervening: Introductory Topics in the Philosophy of Nature, Cambridge, Cambridge University Press.

JOHNSON Mark, (2014), “Experiencing Language: What's Missing in Linguistic Pragmatism?," European Journal of Pragmatism, 6 (2), [http://journals.openedition.org/ejpap/284].

LATOUR Bruno \& Steve WOOLGAR, (1979), Laboratory Life: The Construction of Scientific Facts, Princeton, NJ, Princeton University Press.

MARGOLIS Joseph, (2016), Towards a Metaphysics of Culture, New York, NY, Routledge.

PEREGRIN Jaroslav, (2014), Inferentialism: Why Rules Matter, New York, NY, Palgrave Macmillan.

PRAWITZ Dag, (1965), Natural Deduction: A Proof-Theoretical Study, Stockholm, Almqvist \& Wiksell. Reprinted by Dover Publications, Mineola, NY, 2006.

PRAWITZ Dag, (2006), “Meaning Approached Via Proofs," Synthese, 148 (3), 507-24.

ROUSE Joseph., (2015), Articulating the World, Chicago, IL, University of Chicago Press.

SELLARS Wilfrid, (1956), "Empiricism and the Philosophy of Mind," in Herbert Feigl \& Michael Scriven (eds.), Minnesota Studies in the Philosophy of Science, vol. I, Minneapolis, MN, University of Minnesota Press, 253-329; reprinted in (Sellars 1963), 127-96.

SELLARS Wilfrid, (1961), “The Language of Theories," in Herbert Feigl \& Grower Maxwell (eds.), Current Issues in the Philosophy Science, New York, Henry Holt, Rinehart and Winston, 57-77; reprinted in (Sellars 1963), 106-26.

SELLARS Wilfrid, (1962), "Philosophy and the Scientific Image of Man," in Robert Colodny (ed.), Frontiers of Science and Philosophy, Pittsburgh, PA, University of Pittsburgh Press, 35-78; reprinted in (Sellars 1963), 1-40.

SELLARS Wilfrid, (1963), Science, Perception and Reality, London, Routledge \& Kegan Paul Ltd.

SELLARS Wilfrid, (1975), “The Structure of Knowledge," in Hector-Neri Castañeda (ed.), Action, Knowledge and Reality: Studies in Honor of Wilfrid Sellars, Indianapolis, IN, Bobbs-Merrill, 295-347. TURBANTI Giacomo, (2017), Robert Brandom's Normative Inferentialism, Amsterdam/Philadelphia, John Benjamins Publishing Company.

\section{NOTES}

1. As will be more thoroughly explained in the body of the paper, we take the norms that define a normative space to be expressed and articulated in terms of inferences. However, we do not take a position on the idea that pure and simple inferential rules could make it possible to distinguish between the normative spaces of science and common sense. 
2. The distinction between an empirical and an experimental conception of experience is drawn by Dewey in the 1906 essay Experience and Objective Idealism.

3. For a recent assessment of this issue, see the Symposium Language or Experience. Charting Pragmatism's Course for 21st Century, edited by D. Hildebrand on this journal, Volume 6, Number 2, 2014.

4. This is an acceptable characterization as long as practice and language are correctly conceived. It is important to avoid a possible misunderstanding here. We do not want to convey the idea that practice is not linguistic, or that language is not practical. That view would fly in the face of the overall approach adopted in the present article. Our approach is pragmatist precisely because and insofar as it attempts to take the relation between language and practice seriously: in fact, the very possibility of such relation - although it would be better to talk of 'interrelation' or 'intrinsic relation' - puts some normative constraints on how practice and language should be conceived. They could not enter into relation with one another if they were completely dissimilar. Accordingly, every practice is linguistic through and through, otherwise it would be epistemically irrelevant. At the same time, language is "fraught with oughts," as Sellars put it, in that a discursive practice is needed in order for linguistic expressions to have semantic content.

5. It's worth stressing that the manifest image is only one of the normative spaces in the framework of common sense. Some of these spaces - like e.g. religion, morals or esthetics - have simply nothing to do with science. See below for further discussion.

6. The notion of pragmatic content inverts the relationship between objectivity and representation. 'Pragmatic content' is therefore the technical notion on which our antirepresentationalism relies. For a further discussion of our anti-representationalist approach, see Section 3.

7. In the philosophy of science, the objects of scientific theories are usually referred to as "theoretical entities." Throughout this paper we intentionally refrain from the use of such an expression for two reasons. First, the notion of "theoretical" objects is too often characterized in contrast to the notion of "observational" ones. This opposition, however, is only tangential to our concerns: in fact, we abstain from the use of "observational" as well. Second, if the notion of theoretical objects is not characterized in terms of this opposition (or in terms of other oppositions we are not concerned with here), then it simply amounts to the notion of the object of a theory, which is rather uninformative.

8. When we say that a normative space is required for the definition of a collection of laws we are not committing to any substantial characterization (syntactical, semantical, structural, etc.) of normative spaces. In other words, we do not have a metatheory of normative spaces. Rather, we are interested in the practices that support them.

9. For an analysis of Brandom's normative inferentialism see Turbanti 2017.

10. The seminal idea of an inferentialist semantics is usually traced back to Frege's account of conceptual content ("Begriffliche Inhalt") in the Begriffsschrift (Frege 1879). The very same idea is originally utilized by Carnap (1939) in his logical analysis of syntax. The inferentialist approach to the analysis of the content of logical expressions has been carried out in particular in proof theory. Such a tradition stems out of Gentzen's work (Gentzen 1934-5) and has been developed eminently by Dag Prawitz (see e.g. Prawitz 1965, 2006). Proof-theoretic semantics is a now ongoing enterprise (Francez 2015). In philosophy, inferentialist semantics has been largely investigated in particular by Dummett $(1977,1991)$ and by Brandom $(1994,2000,2008)$. See also Peregrin (2014) for a recent attempt to further develop the subject.

11. It may be worth clarifying that to say that conceptual contents are determined by the norms established in practices is not to say that the content of a concept is the practice that establishes the norms for its use. In other words, the inferentialist semantics that we are presenting is just not representational at all. In particular, it does not deal with truth-makers. However, it is compatible with standard representational semantics (provided that it is used for semantic 
analysis only). So, for instance, while we claim that the content of the SI concept of electron is determined by the norms established in a certain SI practice, we also accept that the truth-maker of a sentence in which the concept of electron is applied is not the bundle of practices that provide the sufficient and necessary conditions for the use of the concept (nor these conditions themselves), but, as one would rightly expect, a certain fact about electrons (or something else, depending on one's theoretical preferences about truth-makers).

12. It is even more so than other possible pragmatist approaches that might be considered as opposing representationalism. The point we would like to stress in this regard is that the idea of problem-solving is not enough to dismantle semantic representationalism. Indeed, one may hold that knowledge is what is achieved at the end of the process of inquiry, and that inquiry is a problem-solving activity; nonetheless, this way of framing the issue - which is clearly antirepresentionalist in the minimal sense that true and justified beliefs do not depict or represent something given before and independently of the inquiry - does not tell anything about how the semantic content of concepts is established. It may well be that the semantic content of the concepts used in the process of inquiry is still defined in purely representationalist terms, even though the purpose of their applications contradicts some of the main assumptions of representationalism. A position of this sort would be a half-hearted anti-representationalism. From this perspective, Dewey's instrumentalist account of concepts counts as a full-blown form of semantic anti-representationalism.

13. The label 'thick' is also used by Brandom to refer to those feedback-governed practices that "essentially involve objects, events and worldly states of affairs" (Brandom 2008: 180; Brandom 2011: 17). In his view, these are practices that cannot be specified without also specifying what they refer to: so, for instance, one cannot specify what hammering is without mentioning hammers and nails. We adopt a thoroughly pragmatist point of view, and by 'thick practices' we mean those practices that involve active coping with the non-linguistic world.

14. As Dewey writes, "[t]he adverb 'truly' is more fundamental than either the adjective, true, or the noun, truth," and he specifies that the reason why the adverb is fundamental is that it "expresses a way, a mode of acting" (Dewey 1920: 182). What Dewey wants to say is that, in order to understand what a concept means, we should look at the practices in which that concept originates and is applied. Clearly, that thesis is a corollary of the pragmatic maxim.

15. The coherence at stake here is not logical consistency: it is a less technical notion, which simply requires that the different operations cooperate towards to a realization of a final end, loosely defined.

16. The third point on this list may seem by far the least important: one may think that it only concerns the socio-political and cultural context in which scientific activity is carried out, that is, something external to the essential core of the SI practice. We disagree. Indeed, we believe that it would sound very strange if a scientist who claims to be an expert in atomic theory were completely ignorant of the impact of her discoveries on scientific communities, as well as of the socio-political bearings that such discoveries may have on the life of his fellow citizens - or, at least, of the possibility that her discoveries may have indirect socio-political consequences. Intuitively, the capacity to locate her work in the different 'institutional' contexts that it may affect is part of what we mean with 'knowing how to use the concept of atom.'

17. On the distinction between instantiate and exemplify, see (Rouse 2015: 295-6).

18. "What is a nomological machine? It is a fixed (enough) arrangement of components, or factors, with stable (enough) capacities that in the right sort of stable (enough) environment will, with repeated operation, give rise to the kind of regular behavior that we represent in our scientific laws." (Cartwright 1999: 50).

19. We are well aware that these remarks only show, at best, that there are some interesting structural similarities between nomological machines and postulational activity in SI. However, we are not committed with a strong identity claim; we are content with the weaker hypothesis 
that they play a similar role in scientific explanation. We leave the issue open for further discussion.

20. The notion of pragmatic metavocabulary is certainly one of the most interesting results of Brandom's meaning-use analysis. To the contrary of what we do here, however, Brandom never considers languages that are pragmatic metavocabularies of themselves. This is because he is mainly interested in the different expressive power of pragmatically related languages, as e.g. logical vocabularies. In this sense, languages like $L_{M}$ and $L_{S}$ and practices like $P_{M}$ and $P_{S}$ are too coarse-grained for his analysis. Notice that pragmatic metavocabularies do not involve paradoxes of self reference like those pointed out by Tarski for semantic metavocabularies.

21. This latter sort of practical elaboration of expressive resources is akin to what Brandom has in mind when he talks about the algorithmic elaboration of new abilities "where exercising the target ability just is exercising the right basic abilities in the right order and under the right circumstances" (Brandom 2008: 26). Of course, this is the basic idea of a computable function in computability theory. Brandom however applies it also to expressive resources. So, for instance, he argues that the ability to deploy conditionals can be algorithmically elaborated from the "primitive abilities to make assertions and to sort inferences into those that are and those that are not materially good ones" (Brandom 2008: 44): in effect, the conditional " $A \rightarrow B$ " can be asserted if and only if the inference from $A$ to $B$ is sorted out as a good one.

22. So, for instance, as far as we can see there is at least another very interesting relation holding from $P_{S}$ to $P_{M}$. Scientific practice in fact may have a deep impact on the practices of common sense through technology. We have not dwelt on technological elaboration in this paper and we defer it to another occasion.

\section{ABSTRACTS}

In our paper we aim to update and revise the pragmatist conception of the relationship between science and common sense. First of all, we introduce two technical notions (MI and SI), with which we identify the normative spaces of the manifest and the scientific image, and we highlight the differences between these two notions and their Sellarsian cognates. Secondly, within each normative space we investigate the connections between languages and practices: we ground linguistic contents on the normative relations that are established in the practices of the corresponding normative space. Finally, we rely on Brandom's meaning-use analysis to provide a representation of the different ways in which MI and SI practices and languages may interact. Our pragmatist proposal is to trace back the ontological conflict that is usually believed to exist between scientific and common sense objects to the differences between scientific and common sense practices.

\section{AUTHORS}

\section{ROBERTO GRONDA}

Università di Pisa

roberto.gronda[at]unipi.it 


\section{GIACOMO TURBANTI}

Università di Trento

turbanti.giacomo[at]gmail.com 